\title{
Electronic properties of graphene nano-flakes:
}

\section{Energy gap, permanent dipole, termination effect and Raman spectroscopy}

\author{
Sandeep K. Singh, ${ }^{1, \text { a) }}$ M. Neek-Amal, ${ }^{1, b)}$ and F.M. Peeters ${ }^{1,(c)}$ \\ Department of Physics, University of Antwerpen, Groenenborgerlaan 171, \\ B-2020 Antwerpen, Belgium
}

(Dated: 6 February 2014)

The electronic properties of graphene nano-flakes (GNFs) with different edge passivation is investigated by using density functional theory. Passivation with $\mathrm{F}$ and $\mathrm{H}$ atoms are considered: $\mathrm{C}_{N_{c}} \mathrm{X}_{N_{x}}(\mathrm{X}=\mathrm{F}$ or $\mathrm{H})$. We studied GNFs with $10<N_{c}<56$ and limit ourselves to the lowest energy configurations. We found that: i) the energy difference $\Delta$ between the highest occupied molecular orbital (HOMO) and the lowest unoccupied molecular orbital (LUMO) decreases with $N_{c}$, ii) topological defects (pentagon and heptagon) break the symmetry of the GNFs and enhance the electric polarization, iii) the mutual interaction of bilayer GNFs can be understood by dipole-dipole interaction which were found sensitive to the relative orientation of the GNFs, iv) the permanent dipoles depend on the edge terminated atom, while the energy gap is independent of it, and v) the presence of heptagon and pentagon defects in the GNFs results in the largest difference between the energy of the spin-up and spin-down electrons which is larger for the H-passivated GNFs as compared to F-passivated GNFs. Our study shows clearly the effect of geometry, size, termination and bilayer on the electronic properties of small GNFs. This study reveals important features of graphene nano-flakes which can be detected using Raman spectroscopy.

PACS numbers: 73.22.-f;71.15.Mb

\footnotetext{
a) SandeepKumar.Singh@uantwerpen.be

b) neekamal@srttu.edu

c) Francois.Peeters@uantwerpen.be
} 


\section{INTRODUCTION}

Graphene nano flakes (GNFs) and graphene nano ribbons (GNRs) are promising graphene based materials with a size controllable energy band gap, which may be useful for different technological applications ${ }^{12}$. In particular, these graphene nano-flakes are important due to their potential for bottom-up fabrication of molecular devices, spintronics and quantum dot technology ${ }^{3}$. Bottom-up and top-down approaches are two alternatives for the production of GNFs. In the first approach, large aromatic hydrocarbons are produced using a large variety of chemical reactions between small molecular units 4 . The top-down method starts with a large piece of graphene sheet and cuts the GNFs out of it. Single graphene sheets can be obtained by a variety of methods, e.g. micromechanical cleavable of a graphite single crystal $^{6}$, starting from graphite oxide ${ }^{7}$ or by chemically unzipping of carbon nanotubes ${ }^{8}$.

Tight-binding (TB) approximation based on the $\pi$ orbitals of carbon, the free massless particle Dirac's equation and ab-initio calculations are three common theoretical methods for studying the electronic and magnetic properties of GNFs and GNRs. Similar to TB calculations ${ }^{9}$ or solutions of the Dirac equation, $\frac{10}{10}$ ab-initio calculations also show that the GNRs have a non-zero direct band gap 11 . Already several studies have appeared on the

electronic and magnetic properties of small GNRs using various methods 12 . However much less extensive and systematic studies are available on various properties of the GNFs, e.g. the size, edge termination and polarization are still poorly understood. Using tight-binding calculation and Hartree-Fock theory the energy gap dependence of triangular and hexagonal GNFs on size, shape and edge were studied by Güçlü et al 13 . It was found that triangular GNFs with zigzag edges exhibit optical transitions in wide spectral ranges, i.e. from teraHertz up to the UV. Because of the small size of the GNFs, they can be considered as a zero-dimensional form of graphene which exhibit very different properties from GNRs and bulk graphene. They are promising for a variety of applications, e.g. electronic and magnetic devices with various molecular sizes and shapes. Graphene nano flakes (graphene quantum dots) can be useful for light absorption relevant for photovoltaics due to their edge structure and wide spectrum. GNFs are found to possess unique electronic, magnetic, and optical properties due to their tunable band gap, e.g., they can be used in solar cells and LED technology 14. They have different corners, mixed zig-zag and arm-chair edges which provide additional degrees of engineering freedom. The small size of GNFs leads to discrete 
energy levels similar to atomic levels in single atoms. The saturation with different atoms or molecular groups on the zigzag edges of rectangular GNFs leads to a spin-polarized ground state with a non zero total magnetic moment, a spin density, and an electronic energy gap that strongly depends on the atomic group used to passivate the dangling bonds ${ }^{15}$. Firstprinciples calculations were also used to investigate the magnetic properties of GNFs with triangular shape and fractal structure ${ }^{16117}$ (see Ref ${ }^{[18}$ for a review).

Only a few studies on the electronic and magnetic properties of GNFs have been published, however most of those studies are limited to triangular or hexagonal shapes of graphene nano flakes with pure zig-zag or arm-chair edges without considering edge passivation, stability $\frac{\sqrt{19}}{}$, polarization effects and melting phenomenon ${ }^{20}$. Here we report on various GNFs with different shapes that are the most energetically favorable configurations for given number of carbon atoms in the flake, as found in our previous work ${ }^{21}$. Such nano flakes were found to have lower melting temperature than graphene ${ }^{20}$. There are few works on bilayer GNFs, e.g. Güçlü et al. ${ }^{22}$ studied graphene bilayer triangular quantum dots and found that it exhibits a shell of degenerate states at the Fermi level. Moreover applying a vertical electric field on bilayer GNF can turn off or reduce the total spin to a single localized spin. In the present study we focus on the electronic properties and permanent polarization of those GNFs with two different edge passivation. We show that the electric dipole moments strongly depend on the symmetry of the GNFs and the type of edge passivation. Our findings for GNFs with $n$-fold symmetry can be extended to larger flakes without loss of generality, they are always un-polarized independent of the type of edge terminated atoms. We also found that the stability of bilayer GNFs depends on the mutual orientation of the permanent dipole moments.

This paper is organized as follows. In Sec. II we give a short review on the most important polarization effects that we need in our study on the GNFs. In Sec. III, we introduce the used DFT calculation. Next in Sec. IV and Sec. V the effects due to symmetry and the energy gap of GNFs and its consequences on the electrical polarization are presented, respectively. The density of states and possible half-metallicity in larger GNFs are also discussed. Sec. VI contains our main results and a discussion on the single sheet GNFs. In Sec. VII we present the results for typical bilayer GNFs and Sec. VIII presents results that are relevant for Raman spectroscopy. Finally, we conclude the paper in Sec. IX. 
(a)

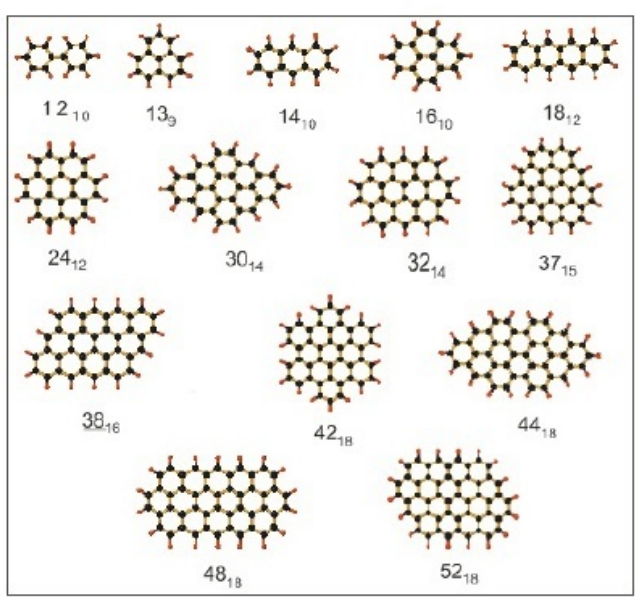

(b)

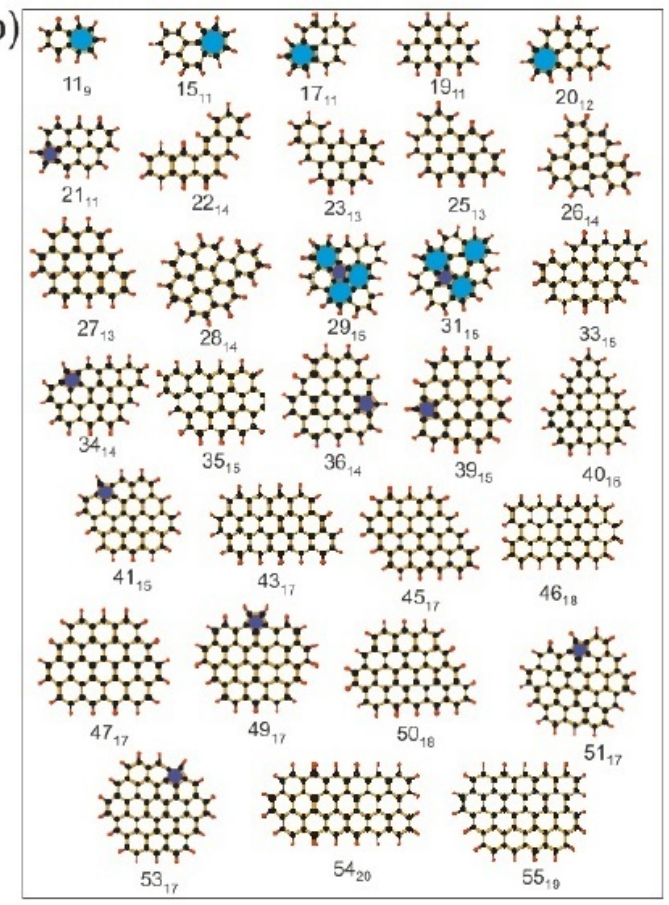

FIG. 1. (Color online) The minimum energy configurations of the studied GNFs. The black balls indicate $\mathrm{C}$ atoms and the red balls can be $\mathrm{H}$ or $\mathrm{F}$ atoms which saturate the edges. (a) The GNFs with n-fold symmetries (the underlined GNFs have 2-fold symmetry). (b) GNFs without n-fold symmetry. The pentagons have indigo color and the heptagons are in blue. The subindex in each GNFs refers to the number of $\mathrm{H}$ or $\mathrm{F}$ atoms and the main number equals the number of $\mathrm{C}$-atoms in the flakes. The shaded polygons are not hexagons.

TABLE I. Binding energy for the three isomers of $\mathrm{C}_{16} \mathrm{X}_{10}$ that are depicted in Fig. 2.

\begin{tabular}{c|c}
\hline & Binding energy $(\mathrm{eV})$ \\
\hline $\mathrm{C}_{16} \mathrm{H}_{10}(\mathrm{a})$ & -170.49 \\
$\mathrm{C}_{16} \mathrm{H}_{10}(\mathrm{~b})$ & -168.10 \\
$\mathrm{C}_{16} \mathrm{H}_{10}(\mathrm{c})$ & -167.78 \\
\hline $\mathrm{C}_{16} \mathrm{~F}_{10}(\mathrm{a})$ & -171.81 \\
$\mathrm{C}_{16} \mathrm{~F}_{10}(\mathrm{~b})$ & -169.47 \\
$\mathrm{C}_{16} \mathrm{~F}_{10}(\mathrm{c})$ & -169.62 \\
\hline
\end{tabular}




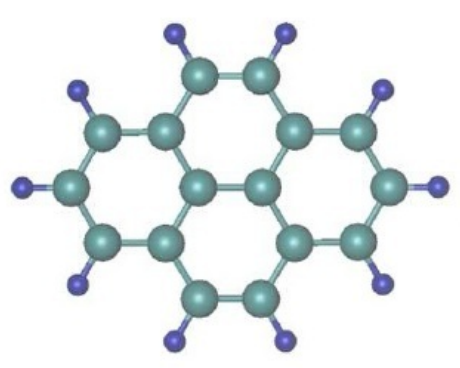

(a)

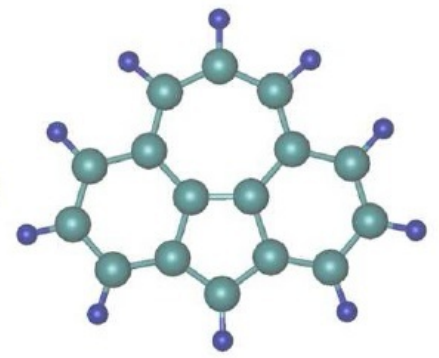

(b)

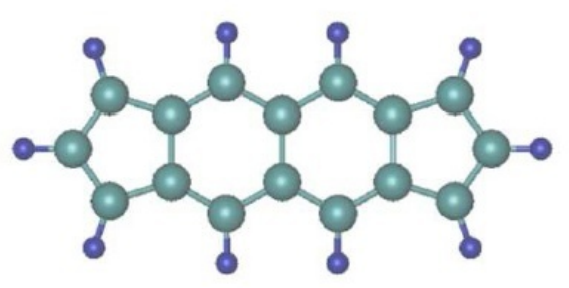

(c)

FIG. 2. (Color online) Three minimum energy isomers of $\mathrm{C}_{16} \mathrm{X}_{10}$ whose energy are given in Table I.

\section{THE COMPUTATIONAL METHOD}

In order to find the electronic dipole moments of the nano flakes we performed DFT calculations on GNFs. We employed density functional theory as implemented in GAUSSIAN (G09) $)^{23}$ which is an electronic-structure package that uses a basis set of Gaussian type of orbitals. For the exchange-correlation (XC) functional, the hybrid B3LYP 24 is adopted in G09, which was shown to give a good representation of the electronic structure in C-based nanoscale systems ${ }^{25}$. Using the polarized basis set $6-311 \mathrm{G}^{* *}$ in G09, we expect that our calculation is capable to provide a reliable description of the electronic properties of the different systems. Notice that for the H-passivated (F-passivated) GNFs if the total number of electrons or equivalently $N_{H}$ e.g. $\mathrm{C}_{21} \mathrm{X}_{11}\left(N_{F}=11\right)$ is an odd number the total spin is non zero and therefore in that case we performed spin polarized calculations.

\section{SYMMETRY EFFECT}

In the case of $\mathrm{H}$-passivation (F-passivation), the passivated $\mathrm{C}$ atoms absorb (gives) part of the electron of the $\mathrm{H}$ (to $\mathrm{F}$ ) atom making the $\mathrm{H}(\mathrm{F})$ atom positively (negatively) charged. 


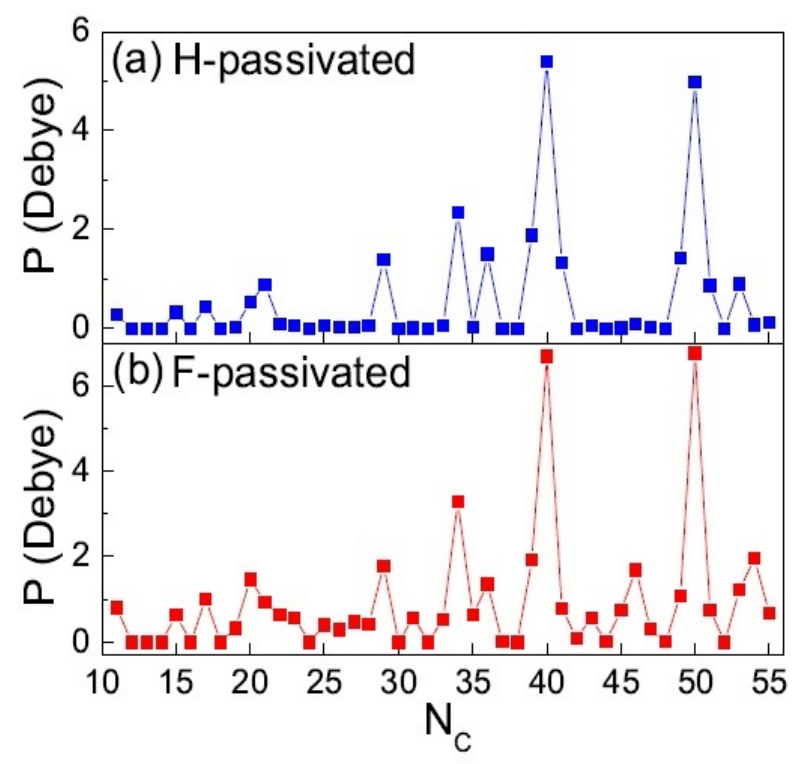

FIG. 3. (Color online) The absolute value of the dipole moment versus the number of carbon atoms for the H-passivated (a) and the F-passivated (b) GNFs. The largest dipoles are for GNFs with $N_{c}=40$ and $N_{c}=50$ in both cases.

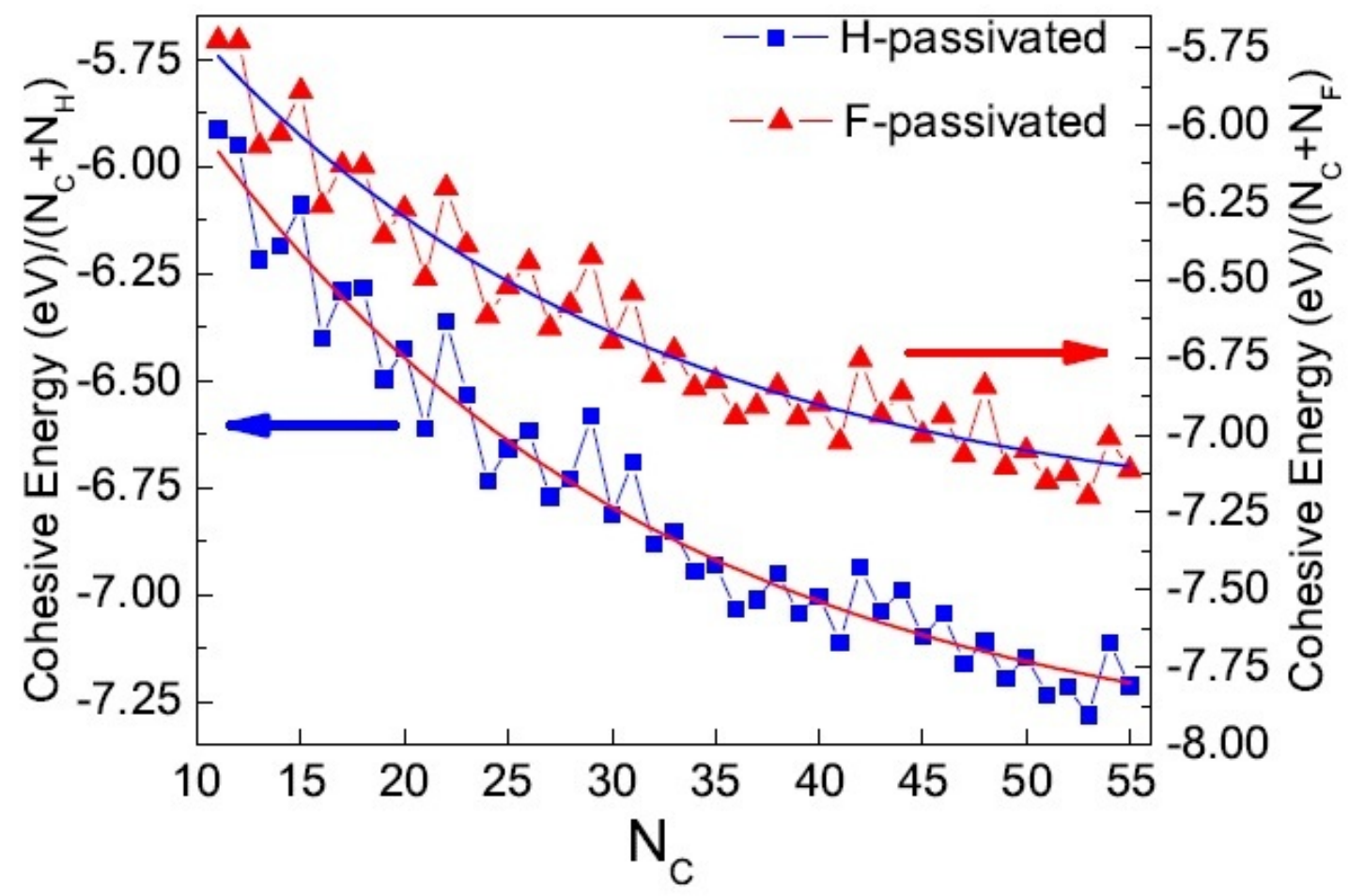

FIG. 4. (Color online) The cohesive energy versus the number of carbon atoms in H-passivated and F-passivated GNFs. The solid lines are the best fits using to Eq.(1). 
This makes the GNFs polarized with a permanent polarization where the net dipole moment is determined from a summation over all local dipoles at the edges. Therefore, the net dipole moment will depend on the geometry of the system which we will study in this section. The electronic and magnetic properties of GNFs originate from this un-balance in charge distribution at the edges.

In Fig. 1 we depict the minimum energy configurations which are saturated by hydrogen or fluorine atoms. These configurations were obtained using the conjugate gradient minimization method as outlined in our previous work ${ }^{211}$ and revisited in the current study by DFT optimization. In order to check the minimum energy configuration we performed extra calculations for three typical isomers with $\mathrm{N}_{C}=16$ (see Fig. 2). The corresponding binding energy are listed in Table I. The lowest energy configuration is the $16_{10}$ structure in Fig. 1. The defective $\mathrm{H}$ - and F-passivated small clusters are slightly buckled after relaxation. We categorized the studied GNFs into two different groups: i) fourteen GNFs with $n$-fold symmetry, e.g. $\mathrm{C}_{12} \mathrm{H}_{10}$ with $n=2, \mathrm{C}_{13} \mathrm{H}_{9}$ with $n=3$ and so on, which are shown in Fig. 1(a); and ii) the systems with pentagon and heptagon defects (shaded) and those without $n$-fold symmetry, e.g. $\mathrm{C}_{11} \mathrm{H}_{9}$ which are shown in Fig. $1(\mathrm{~b})$. The latter systems may have mirror symmetry. Here we limit our study to those $\mathrm{C}_{N_{c}} \mathrm{X}_{N_{x}}$ structures that have minimum energy for planar flakes. The first aforementioned group has always zero dipole moment while the second group has non-zero total dipole moment and in some cases are even a giant polar molecule. Notice that larger flakes with n-fold symmetry (the first group) should also have zero total dipole moment.

In the first group (Fig. 1(a)), e.g. in the 2-fold symmetry cases a rotation of $180^{\circ}$ around the z-axis transforms $\mathbf{r}_{i} \rightarrow-\mathbf{r}_{i}$ and consequently $\vec{P}_{T}=0$. In the second group there is no n-fold symmetry where different edges have different orientation of local dipole moments which do not cancel each other hence resulting in a non-zero net dipole moment. More defects that are randomly distributed increase the dipole moment strength.

In Fig. 3 we show the absolute value of the total dipole moment versus $N_{c}$ for all studied GNFs. In Fig. 3(a) the H-passivated system and in Fig. 3(b) the F-passivated system are shown. We see that in both cases the above mentioned symmetry issues are obeyed. The net dipole of GNFs with F-passivation are larger than those for H-passivation which is due to the larger electronegativity of $\mathrm{F}$. It is interesting to note that the two systems with mirror symmetry, i.e. $\mathrm{C}_{40} \mathrm{H}_{16}$ and $\mathrm{C}_{45} \mathrm{H}_{17}$ have respectively the largest - 6.35 Debye - and 


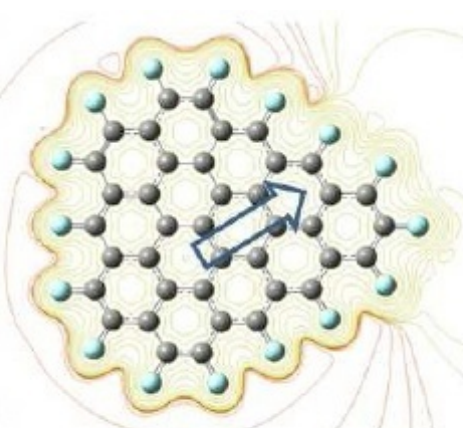

(a)

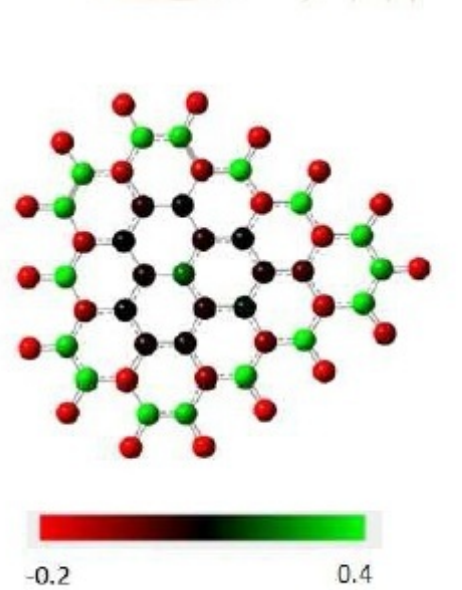

(b)

(c)

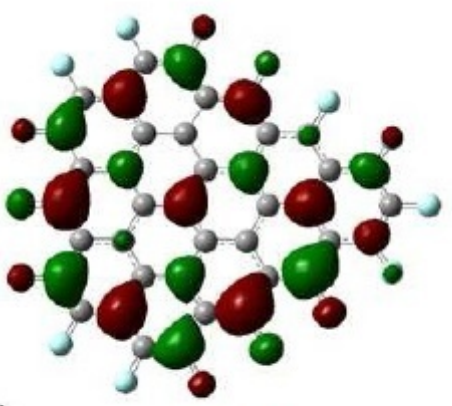

HOMO

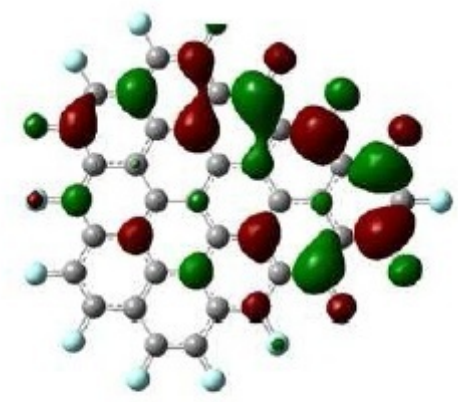

(d)

LUMO

FIG. 5. (Color online) (a) Electrostatic potential contours around F-passivated GNFs - $\mathrm{C}_{40} \mathrm{~F}_{16^{-}}$ which has the largest dipole moment indicated by the arrow, (b) Corresponding charge distribution, (c) HOMO and (d) LUMO orbitals. The red and green colors stand for the positive and negative signs of the molecular orbital wave function, respectively.

the smallest - 0.015 Debye - dipole moment. The corresponding dipole moment for $\mathrm{C}_{40} \mathrm{~F}_{16}$ and $\mathrm{C}_{45} \mathrm{~F}_{17}$ are 7.69 Debye and 0.61 Debye, respectively. Notice that the larger component of the dipole moment is along the symmetry axis. The system $\mathrm{C}_{50} \mathrm{X}_{18}$ without particular symmetry has the second largest dipole moment. The ratio between the dipole moments of $\mathrm{C}_{40} \mathrm{~F}_{16}$ and $\mathrm{C}_{40} \mathrm{H}_{16}$ is 1.21 .

It is surprising that a simple linear summation over all local dipole moments in e.g. $\mathrm{C}_{40} \mathrm{X}_{16}$, apparently leads to a zero dipole moment if one assumes equal $\vec{p}_{C-X}$ for all saturated bonds, while this cluster has the largest dipole moment. The reason for such effect is that the local dipole moments $\vec{p}_{C-X}$ are not in the same direction due to the non-uniform distribution of the C-X second neighbors, i.e. most of the $\mathrm{C}$ atoms in the bottom of $\mathrm{C}_{40} \mathrm{X}_{16}$ (see Fig. 1(b)) are connected to the inner $\mathrm{C}$ atoms but at the top of the system there is no 
inner $\mathrm{C}$ atom to give more electron charge to the corresponding $\mathrm{C}-\mathrm{X}$ bonds.

\section{ENERGY DIFFERENCE BETWEEN HOMO AND LUMO}

We study small carbon flakes that are saturated by $\mathrm{H}$ or $\mathrm{F}$ which have discrete energy levels and are filled by electrons. In the case of spin polarized calculations there is an extra energy level. In Fig. 4 we plot the cohesive energy versus $N_{c}$ for all studied GNFs. It is seen that the energy per atom decreases with $N_{c}$ in both H-passivated and F-passivated GNFs. Increasing the number of $\mathrm{C}$ atoms in GNFs increases the number of $\mathrm{C}-\mathrm{C}$ bonds more rapidly than the C-X bonds at the edges. Since the total cohesive energy is a function of C-C and C-X binding energy we expect that the total cohesive energy rapidly approaches the bulk cohesive energy $\left(e_{0}\right)$. On average we found that the energy decreases according to

$$
E /\left(N_{c}+N_{X}\right)=e_{0}+b e^{-\lambda N_{c}}
$$

where $e_{0}=-7.4 \mathrm{eV}, \mathrm{b}=-2.37 \mathrm{eV}$ and $\lambda=0.045$ for $\mathrm{H}$-passivated and $e_{0}=-7.3 \mathrm{eV}, \mathrm{b}=-2.53 \mathrm{eV}$ and $\lambda=-0.046$ for F-passivated GNFs which are shown by the solid curve in Fig. 4. Because the $\mathrm{C}-\mathrm{F}$ binding energy is larger than $\mathrm{C}-\mathrm{H}$ in $\mathrm{F}$ saturated GNFs the curve for F-passivated is above the one of $\mathrm{H}$-passivated. For larger $\mathrm{N}_{X} / \mathrm{N}_{C}$ ratio the total cohesive energy is far from the bulk energy which is due to the so called, i.e. edge effect. Adding a $\mathrm{C}$ atom to a typical GNF decreases the energy if the new formed GNF becomes more stable which is mostly the case for those GNFs without pentagon and heptagon defects. If the next GNF has pentagon or heptagon defects the energy is larger, see e.g. $N_{c}=11,15,17,20,29,31$. We emphasize that most of the studied GNFs are planar-like structures. Moreover the systems

with a larger ratio $\frac{N_{c}-N_{x}}{N_{x}}$ have lower energy which corresponds to higher stability due to the lower number of edge atoms. It is seen that $\Delta E=E_{N_{c+2}}-E_{N_{c}}=$ constant for some $N_{c}$ (e.g. between $N_{c}=42$ and $N_{c}=53$ ) where $\Delta N_{H}=0$ in H-passivated GNFs. This is promising for quantum dot design: fixing the number of edge $\mathrm{H}$-atoms and increasing the number of $\mathrm{C}$ atoms decreases the energy of the system with constant increments. The latter effect is similar to the regular jumps observed in the electrochemical potential in quantum dots. First we compare the energy of two typical GNFs ( with only hexagons, e.g. $\mathrm{C}_{37} \mathrm{X}_{15}$ ) and with also pentagon and heptagon, e.g. $\left.\mathrm{C}_{39} \mathrm{X}_{15}\right)$ with the same flakes without $\mathrm{X}$-atoms at the edges (bare flakes). Results show that in both cases independent of the type of X atom 
edge passivation the total energy is reduced, i.e. $\mathrm{E}\left(\mathrm{C}_{37} \mathrm{~F}_{15}\right)-\mathrm{E}\left(\mathrm{C}_{37}\right)=-1.9948 \mathrm{eV}, \mathrm{E}\left(\mathrm{C}_{37} \mathrm{H}_{15}\right)$ $\mathrm{E}\left(\mathrm{C}_{37}\right)=-1.9380 \mathrm{eV}, \mathrm{E}\left(\mathrm{C}_{39} \mathrm{~F}_{15}\right)-\mathrm{E}\left(\mathrm{C}_{39}\right)=-1.9298 \mathrm{eV}$, and $\mathrm{E}\left(\mathrm{C}_{39} \mathrm{H}_{15}\right)-\mathrm{E}\left(\mathrm{C}_{39}\right)=-1.8724 \mathrm{eV}$. The energy decreases much more in $\mathrm{C}_{37} \mathrm{X}_{15}$ than for $\mathrm{C}_{39} \mathrm{X}_{15}$. Therefore the GNFs with regular hexagon edges becomes more stable when they are saturated by $\mathrm{X}$ atoms with respect to the GNF which contain heptagons and pentagons. This is in agreement with those reported for triangular GNFs flakes in Ref. 17.

In Fig. 5 we show for $\mathrm{C}_{40} \mathrm{~F}_{16}$ (a) the electrostatic contour, (b) charge distribution, (c) the corresponding HOMO and (d) LUMO. The arrow in (a) indicates the giant permanent dipole in $\mathrm{C}_{40} \mathrm{~F}_{16}$. There is a clear relation between the electrostatic potential and the charge distribution. The LUMO is localized on the parts with transferred charges to the HOMO region. The less symmetry in any GNFs leads to the non-horizontal direction of the dipole moment. The dipole moment is directed to the region with the LUMO orbitals.

In all n-fold symmetric GNFs the net dipole is zero while one can define local dipoles which eventually cancel each other. Note that using a different functional for the exchange correlation in our DFT calculation may change the energy gap slightly, however the energy gap for H-passivated and F-passivated GNFs are close to each other. This is in agreement with the results of Ref. [15].

In Fig. 6 we show the electrostatic contour lines around $\mathrm{C}_{41} \mathrm{~F}_{16}$ (a) which has non-zero total spin. The corresponding spin-up and spin-down HOMO and LUMO are shown in (b). There is a clear difference in the orientation between orbitals of spin-up and spin-down which results in a different energy gap between spin-up and spin-down electrons. The spinup electrons have larger energy gap as compared to spin-down. Notice that we do not show the HOMO and LUMO for all studied GNFs which can be made available upon request. As a typical case, the isosurface of the spin density for $\mathrm{C}_{41} \mathrm{~F}_{16}$ is shown in Fig. 6(c). Although the frontier orbital are mostly nonuniformly distributed at the edges, the isosurface of the spin density are uniform except on the heptagon's atoms, i.e. only the $\alpha$ spin has a considerable value different from zero. In general for a graphene nanoflakes with zigzag edges, the spin magnetizations of $\mathrm{A}$ and $\mathrm{B}$ sublattices are aligned antiparallel, i.e. antiferromagnet spin ordering $\frac{16}{16}$ which is not similar to our studied GNFs. This alternative is broken by the defect in a way that depend on the out-of-plane distortion ${ }^{26}$.

In Fig. 7 we show the HOMO-LUMO energy gap $\Delta$ versus $N_{c}$ which on average decreases linearly up to $N_{c} \sim 35$. The larger the electrostatic potential difference between the ends 
of GNFs, the lower the energy gap. The electrostatic potential due to the local charge transfer between $\mathrm{C}$ and $\mathrm{X}$ decreases the energy of the LUMO level and increases the energy of the HOMO level thus reducing the energy gap. We fit a linear line to the results for both H-passivated and F-passivated systems. This curve is shown in Fig. 7. For large GNFs $\left(N_{c}>35\right)$ the energy gap is almost constant. It is well known that the energy gap of graphene is zero. Tight binding calculations predict that in hexagonal (triangular) GNFs having more than $10^{3}\left(10^{6}\right) \mathrm{C}$ atoms the energy gap approaches zerd $\frac{13}{}$. In finite size GNFs the energy gap strongly depends on the geometry of the system and the type of edges, e.g. zigzag versus armchair. we found in previous work that the energy gap decreases like a/N where $\mathrm{a}=4.9 \mathrm{eV} 27$. On the other hand experiments on GNFs revealed that the energy gap decreases like $1 / \mathrm{L}$ where $\mathrm{L}$ is the lateral dimension of GNF 28129 . Here our studied GNFs typically have no particular edge structure and we found that the energy gaps decrease up to about $2 \mathrm{eV}$.

Although the polarization of GNFs can be understood from the possible symmetries of GNFs, however the difference between the energy of the HOMO and the LUMO $(\Delta)$ can not be explained simply as due to an increase of the dipole moment, i.e. the energy gap is

a scaler physical quantity and one does not expect that all systems with $\vec{P}_{T}=0$ have zero energy gap. In other words there is no straightforward relation between the symmetry and the energy gap in GNFs. The energy gap is affecten by the non-uniform distribution of the electrostatic potential which is a function of the non-uniform charge distribution over the GNFs. Nevertheless as seen from Figs. 3 and 7 the GNFs with the largest dipole moment have the lowest energy gap hence the polar GNFs in this study have a lower energy gap which originate from the un-balanced charge distribution. This effect strongly depends on the geometry and can not be generalized to every isomer with the same number of $\mathrm{C}$ atoms.

\section{DENSITY OF STATES AND SPIN POLARIZED RESULTS}

In Fig. 8 we show the density of states (DOS) for those systems with the largest dipole moment, i.e. $\mathrm{C}_{40} \mathrm{X}_{16}$ (zero energy gap $\Delta$ ) and $\mathrm{C}_{34} \mathrm{X}_{14}$ with energy gap equal to $1.48 \mathrm{eV}$ for H-passivated and $1.42 \mathrm{eV}$ for F-passivated. We set the Fermi energy at zero by defining $E_{F}=\left(E_{\text {HOMO }}+E_{L U M O}\right) / 2$. Although the energy gap is almost zero in GNFs with $N_{c}=$ 40, 50 but there is a significant gap between the $\mathrm{HOMO}, \mathrm{HOMO}_{2}$ and $\mathrm{LUMO}$ and $\mathrm{LUMO}_{2}$ 


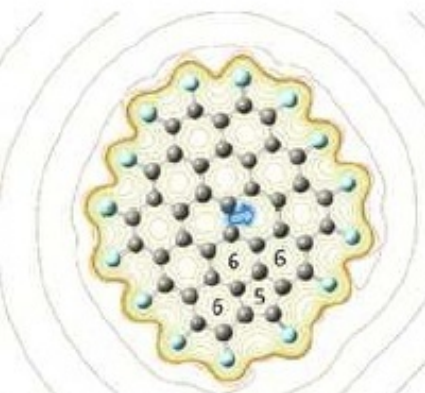

(a)

(c)

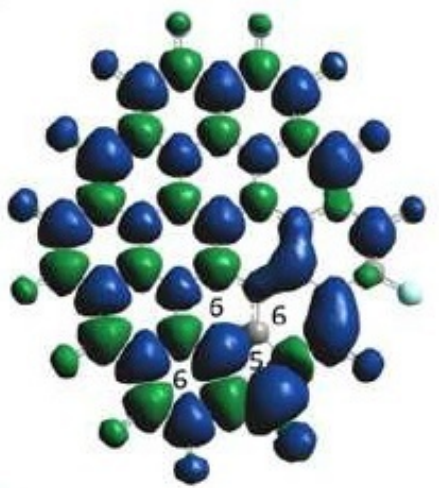

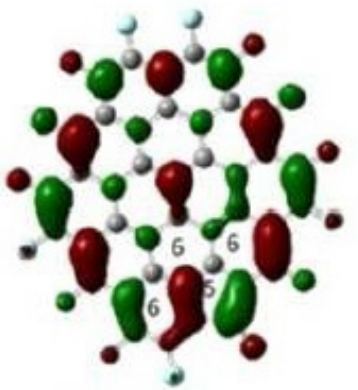

$\mathrm{HOMO}$ t

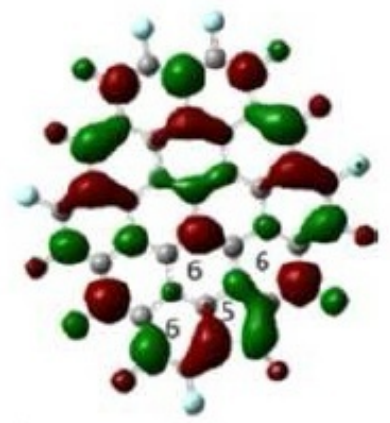

(b) LUMO 1

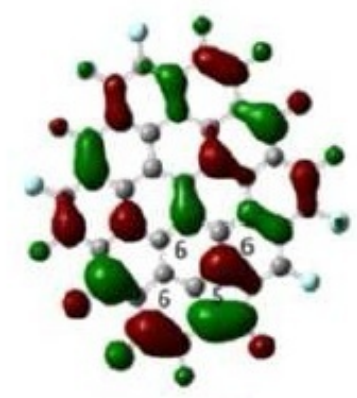

$\mathrm{HOMO} \downarrow$

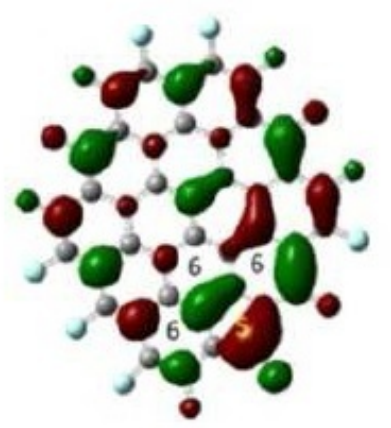

LUMO

FIG. 6. (Color online) Electrostatic potential contours around $\mathrm{C}_{41} \mathrm{~F}_{15}$ with non zero total spin. The dipole moment is indicated by the arrow. (b) The corresponding spin-up and spin-down HOMO and LUMO orbitals. The red and green colors stand for the positive and negative signs of the molecular orbital wave function, respectively. (c) Isosurfaces of spin density for $\mathrm{C}_{41} \mathrm{~F}_{15}$. Blue and green isosurfaces are $0.0004 \mathrm{e} / \AA^{3}$ and $-0.0004 \mathrm{e} / \AA^{3}$, respectively.

which results in larger ionization energy for the electrons in HOMO2, where index 2 refers to the closest occupied state to HOMO with larger energy than HOMO. Furthermore the occupied states in F-passivated GNFs have larger DOS as compared to H-passivated GNFs which is attributed to the larger charge transfer to the system by $\mathrm{F}$ atoms than $\mathrm{H}$ atoms.

It is also interesting to investigate the DOS of systems with non-zero total spin. In Fig. 9 the DOS of $\alpha$ (spin-up: top panels with black color) and $\beta$ (spin-down: bottom panels with red color) spins are shown for three typical systems, i.e. $\mathrm{C}_{51} \mathrm{X}_{17}, \mathrm{C}_{41} \mathrm{X}_{15}$ and $\mathrm{C}_{35} \mathrm{X}_{15}$. The spin-up and spin-down DOS are almost symmetrical except around the gap region. There is a clear difference between Figs. 9(a) and 9(b) (and also (c)) while the number of X atoms are the same, $N_{x}=15$. In order to find the Fermi energy we sorted all the energy 


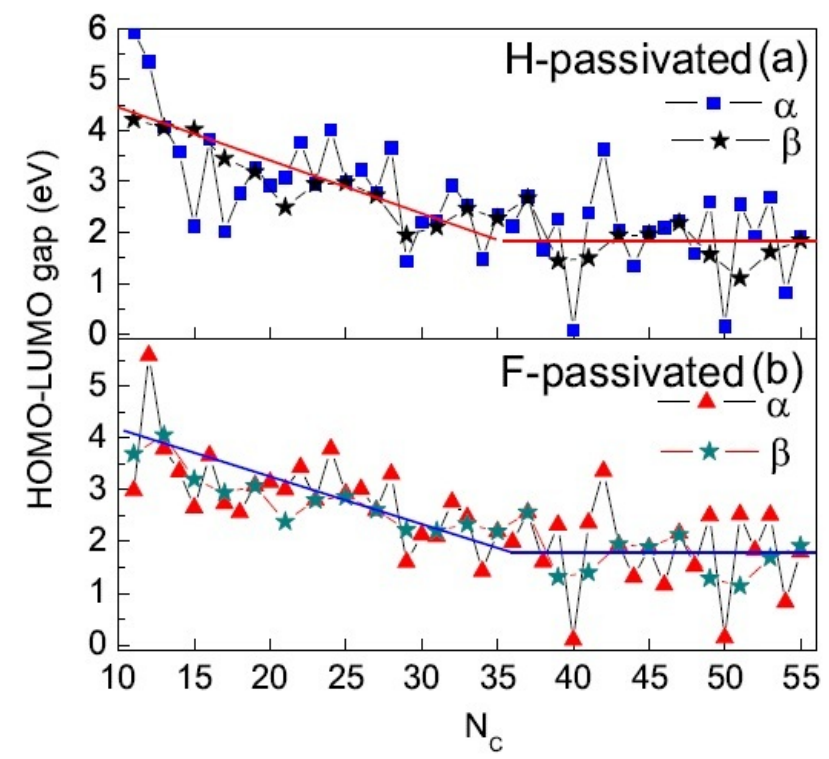

FIG. 7. (Color online) HOMO-LUMO gap versus the number of carbon atoms in H-passivated (a) and F-passivated GNFs(b). The gap approaches zero and is close to zero beyond $N_{c}=35 . \alpha$ and $\beta$ stand for the HOMO-LUMO gap for spin up and spin down, respectively. The solid lines show the overall change in the gaps.

levels for spin-up and spin-down and we found the middle point of the new HOMO and LUMO. We found that the energy gaps between the spin-up and spin-down electrons are different. In Fig. 10 we show the absolute value of the difference between the energy gap of $\alpha$ spin and $\beta$ spin, i.e. $\Delta_{\alpha}-\Delta_{\beta}$. It is surprising that the presence of pentagons in GNFs with non-zero total spin maximize $\Delta_{\alpha}-\Delta_{\beta}$. The largest difference is for $N_{c}=15$ and $N_{c}=51$ which have one heptagon and one pentagon, respectively. The symbols ' 5 ', ' 6 ' and ' 7 ' in Figs. 10(a,b) indicate the presence of pentagon, only hexagons and heptagon in the corresponding GNFs. Notice that the H-passivated GNFs have a larger difference between the energy gap of spin-up and spin-down electrons however the overall pattern in Fig. 10 is the same. Using these results one can predict that for large GNRs with a few pentagons and/or heptagons half-metallicity can be found.

The GNRs exhibit half-metallicity in the presence of electric field ${ }^{3}$. It was found that the half-metalicity in GNRs originates from the fact that the applied electric field induces an energy level shift of opposite sign for the spatially separated spin ordered edge states. 

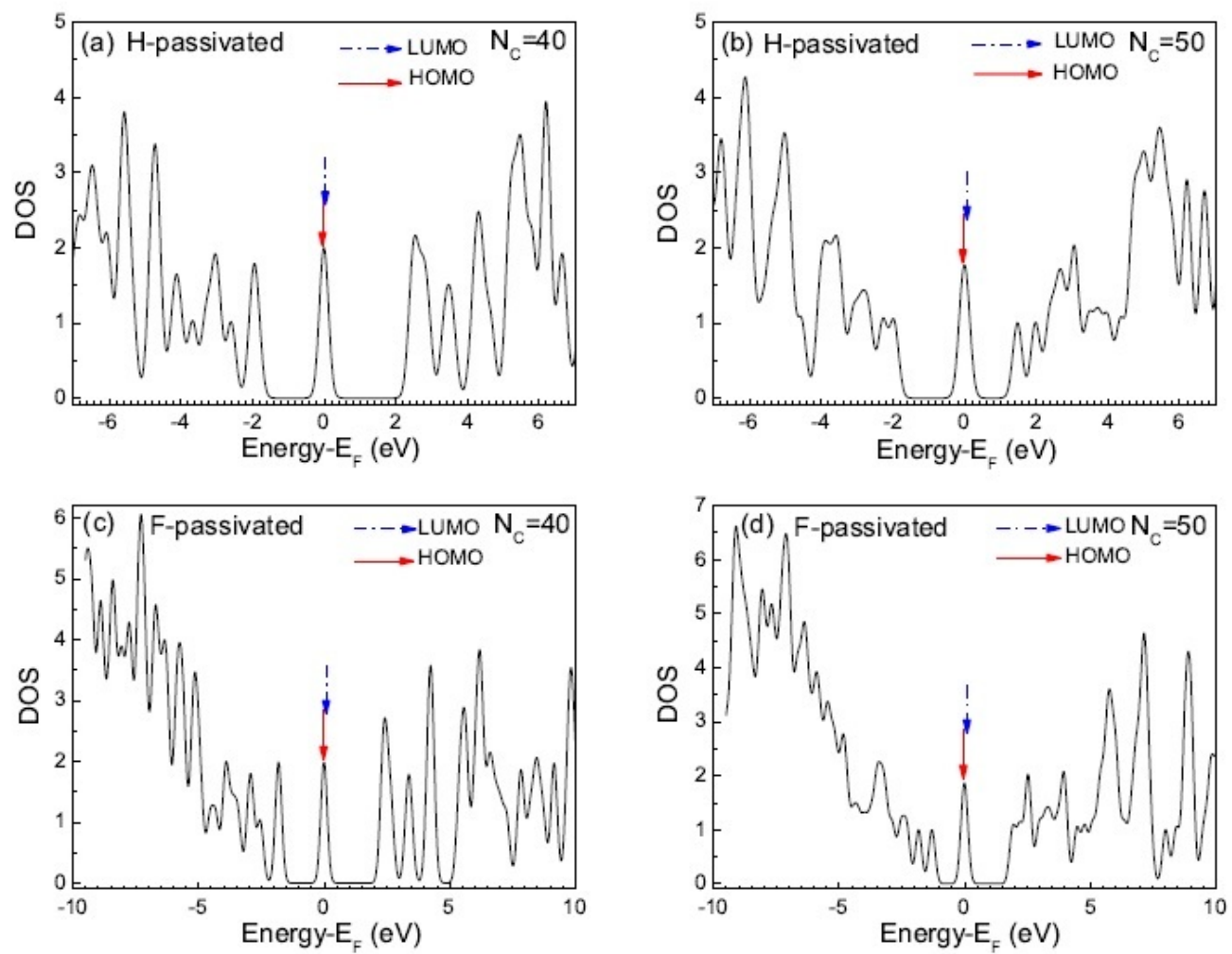

FIG. 8. (Color online) Density of states for H-passivated (a,b) and F-passivated (c,d) graphene nano flakes; the energy gap in $(\mathrm{a}, \mathrm{b}, \mathrm{c}, \mathrm{d})$ is almost zero while they have maximum dipole moment among the studied GNFs. The vertical arrows refer to the position of the HOMO (red) and LUMO (blue). The Fermi energy was set at zero.

\section{BILAYER GNFS $\left(\mathbf{C}_{40} \mathbf{H}_{16}\right.$ AND $\left.\mathbf{C}_{40} \mathbf{F}_{16}\right)$}

Bilayer GNFs, which to the best of our knowledge, have not been investigated from first principles. We select GNFs which have the largest dipoles. In order to study the bilayer GNFs we performed three typical DFT calculations for two bilayer $\mathrm{GNFs}_{40} \mathrm{H}_{16}$ and three other DFT calculations $\mathrm{C}_{40} \mathrm{H}_{16}$ which have different mutual orientation which is characterized by rotation angle with respect to each other $\theta$, i.e. parallel with $\theta=0^{\circ}$, (Fig. 11)(a)), parallel with $\theta=180^{\circ}$ (Fig. 11(e)) and parallel with $\theta=90^{\circ}$ (Fig. 11(i)). For the case $\theta=0^{\circ}, 180^{\circ}$ GNFs in adjacent layers are arranged head-to-head (and tail-to-tail) and head-to-tail arrangement, respectively.

i) $\theta=0^{\circ}$ : In Figs. 11(a)(b) we show a side and top view, respectively, of the optimized 

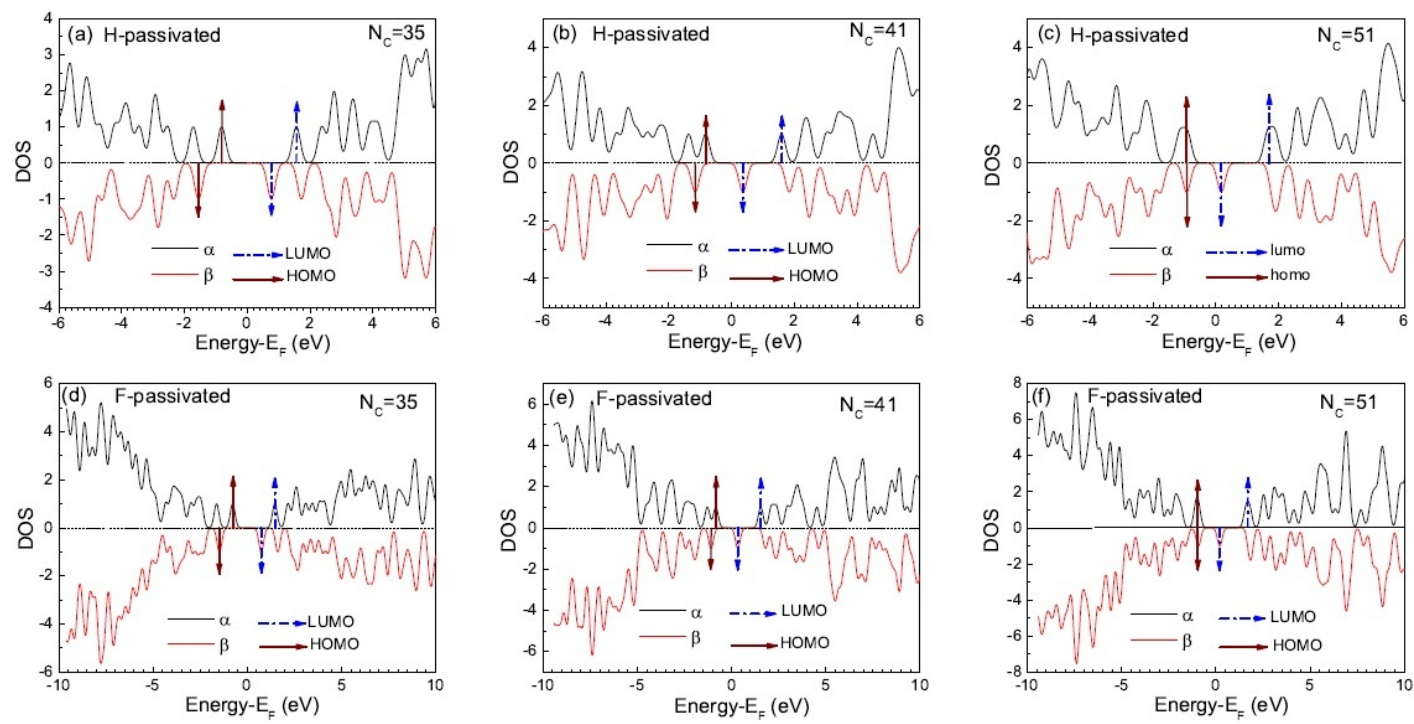

FIG. 9. (Color online) Density of states for H-passivated (a,b,c) and F-passivated (d,e,f) graphene nano flakes with non-zero total spin; the energy gap in (b,c,e,f) are different for spin-up (denoted by $\alpha$ ) and spin-down (denoted by $\beta$ ) electrons with a shift in Fermi energy with respect to each other. In $(\mathrm{a}, \mathrm{d})$ the energy gap for spin-up and spin-down are almost the same with a shift in Fermi energy with respect to each other. The Fermi energy occurs at zero.

structure for two bilayer $\mathrm{C}_{40} \mathrm{H}_{16}$ with $\theta=0^{\circ}$ which after optimization are found to be bent with positive curvature. GNFs of $\mathrm{C}_{40} \mathrm{H}_{16}$ in the adjacent layers are directed head-to-head and tail-to-tail. The closest and longest distance between two adjacent layers (not including the hydrogen atoms) are found to be 3.27 and $4.83 \AA$, respectively, which are closer and longer than the distance between graphite layers, i.e. $3.35 \AA$. The optimized structure indicates charge repulsion in the ends (tails) in bilayer GNFs. Because $\mathrm{C}_{40} \mathrm{H}_{16}$ is a giant polar molecule, we can understand the repulsion of two bilayer molecules as the dipole-dipole interaction, i.e. $U=\vec{P}_{1} \cdot \vec{P}_{2} / R^{3}$ where $R$ is the perpendicular distance between two dipoles. If the sheets do not bent we expect that $\theta=180^{\circ}$ has lower energy than $\theta=0^{\circ}$, however bending reduces the energy and makes both energies close to each other. In Table II we listed all results for bilayer $\mathrm{C}_{40} \mathrm{H}_{16}$ and $\mathrm{C}_{40} \mathrm{~F}_{16}$.

The HOMO-LUMO and DOS for $\theta=0^{\circ}$ are illustrated in Figs. 11(c,d) and Figs. 12 (a), respectively. There is a clear mixing of orbitals between two HOMOs in adjacent GNFs (see Figs. 11(c)). This is different from the stacking of graphite where the layers are parallel 


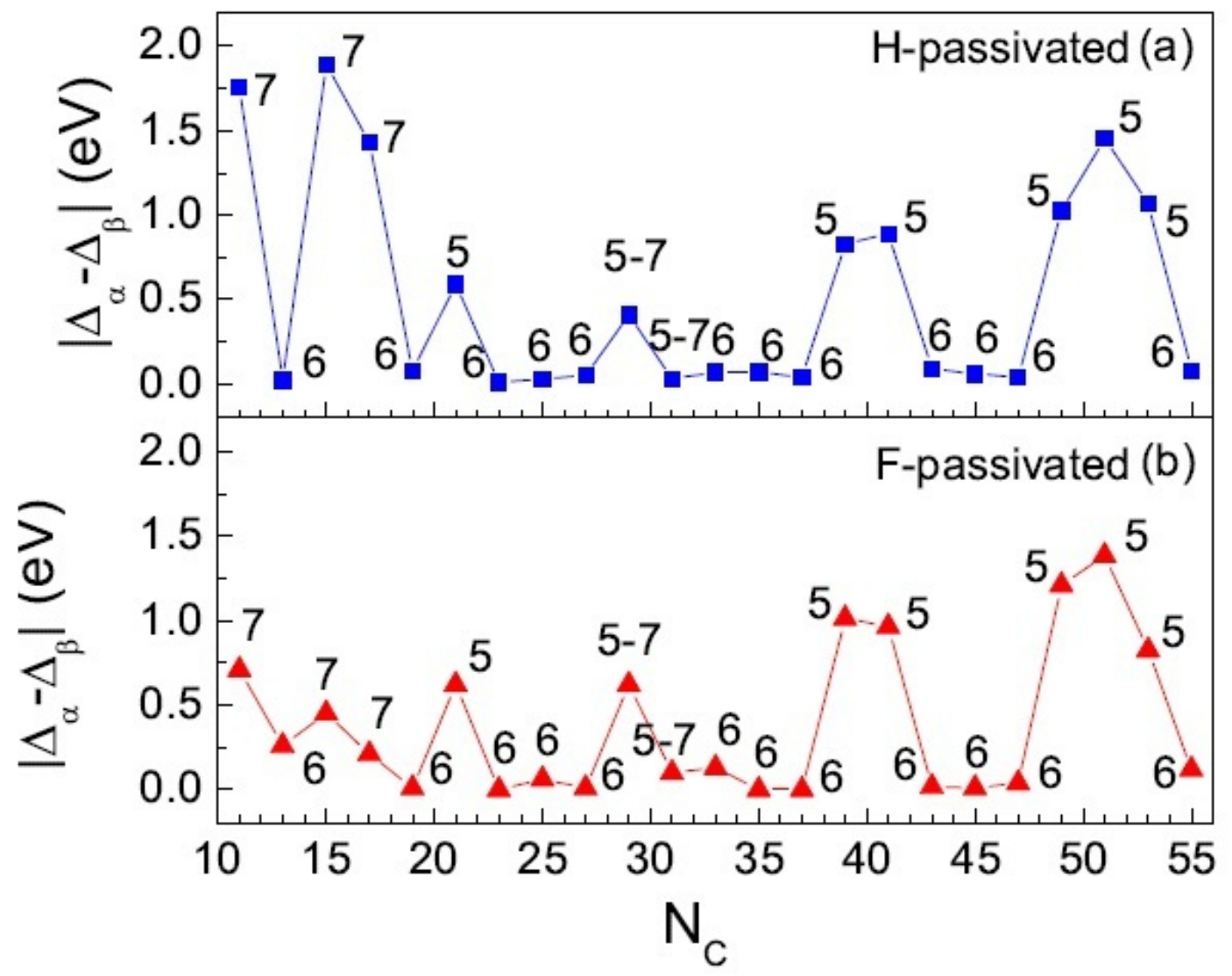

FIG. 10. (Color online) The energy gap between spin up and spin down for H-passivated (a) and F-passivated (b) graphene nanoflakes. The numbers refer whether there are pentagon (5), hexagon (6) or heptagon (7) in the corresponding GNF.

and there is no electron-sharing between two layers and the interaction is mostly a weak vander Waals interaction. This mixing effect reduces the energy, otherwise we expect that the $\theta=0^{\circ}$ case has higher energy than $\theta=180^{\circ}$. The HOMO-LUMO gap $0.97 \mathrm{eV}$ appears in bilayer GNFs which was found to be almost zero for a single $\mathrm{GNF} \mathrm{C}_{40} \mathrm{H}_{16}$, see Fig. 7 . Now there is a small gap between the HOMO, $\mathrm{HOMO}_{2}$ and $\mathrm{LUMO}$ and $\mathrm{LUMO}_{2}$ in contrast to single $\mathrm{GNF} \mathrm{C}_{40} \mathrm{H}_{16}$.

ii) $\theta=180^{\circ}$ : For this case the head of the top GNF is directed towards the tail of the bottom GNF. The optimized structure leads to a relative rotation, i.e. the optimized structure does not satisfy the condition $\theta=180^{\circ}$. Here curvature is negative and the closest distance between different layers (not including hydrogen atoms) thus appears in the vicinity 


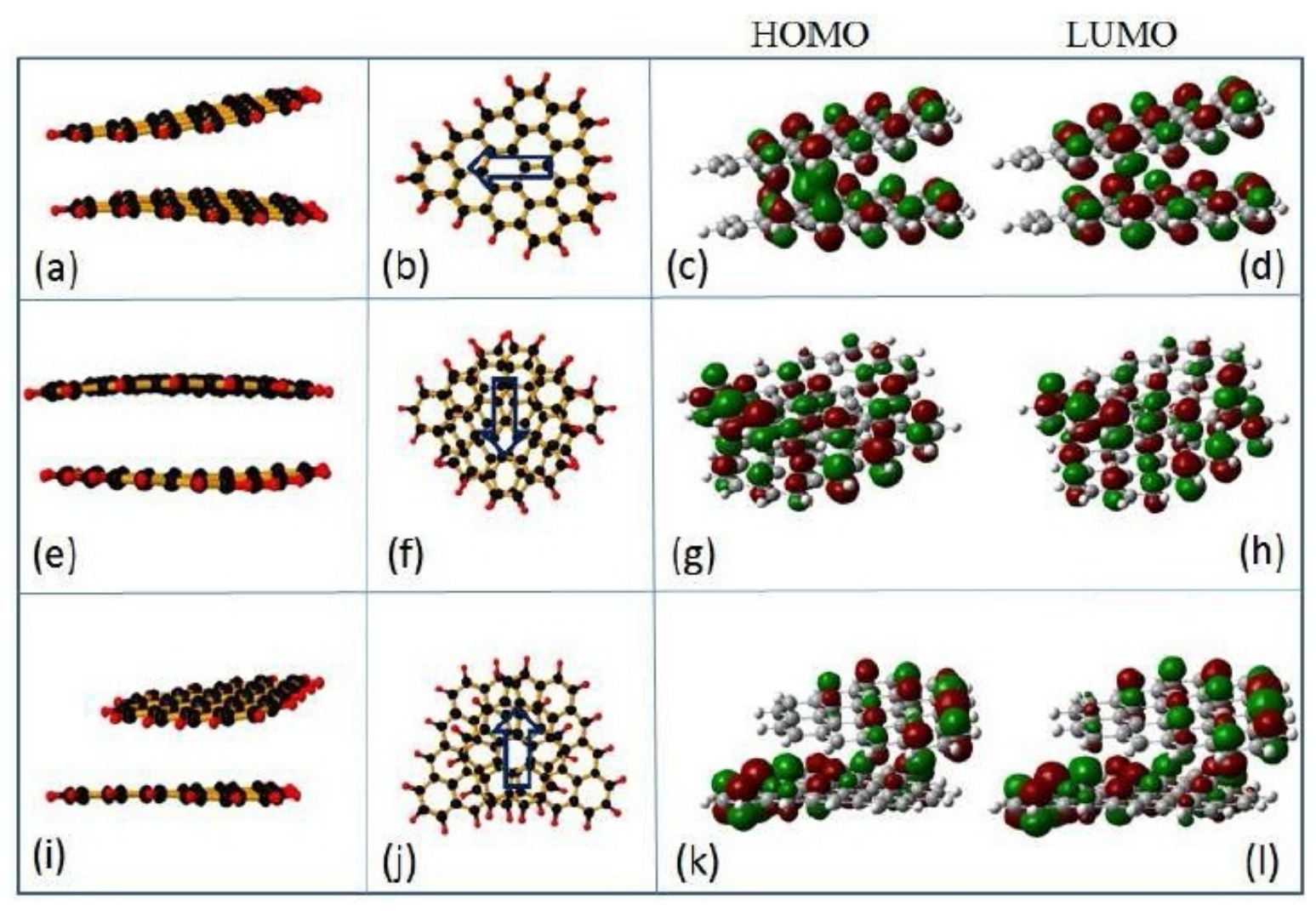

FIG. 11. (Color online) Bilayer GNFs $\mathrm{C}_{40} \mathrm{~F}_{16}$ : parallel with $\theta=0^{\circ}$ (a) side view (b) top view (c) HOMO (d) LUMO; parallel with $\theta=180^{\circ}$ (e) side view (f) top view (g) HOMO (h) LUMO; parallel with $\theta=90^{\circ}$ (i) side view (j) top view (k) HOMO (l) LUMO. The arrows in Figs.(b,f,j) indicate the direction of polarization.
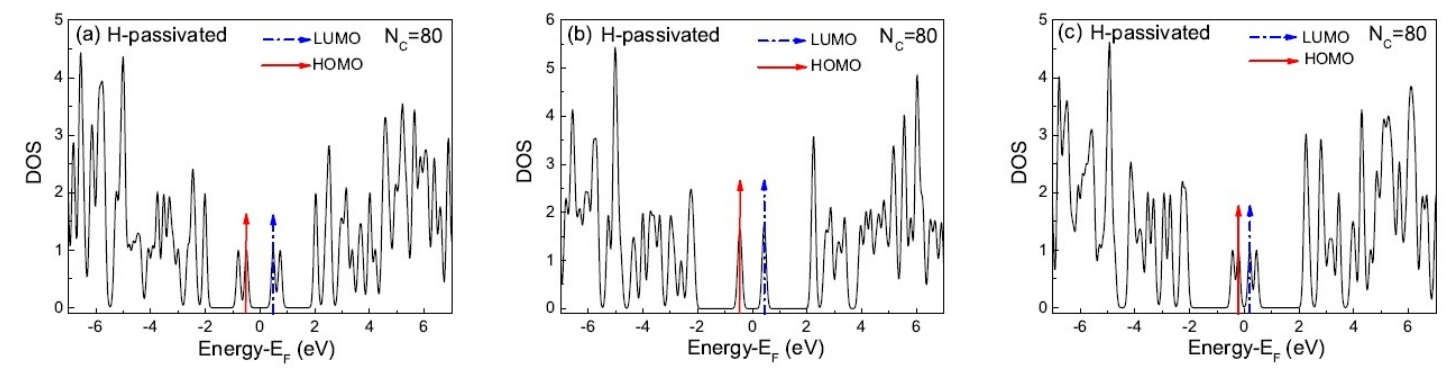

FIG. 12. (Color online) Density of states for H-passivated bilayer GNFs (a) parallel with $\theta=0^{\circ}$ (b) parallel with $\theta=180^{\circ}$ (c) parallel with $\theta=90^{\circ}$. An energy gap appears in contrast to almost zero gap in single GNF $\mathrm{C}_{40} \mathrm{H}_{16}$. The vertical arrows refer to the position of the HOMO (red) and LUMO (blue). 
of these head-tail, and the distance of the head1-tail2 and tail1-head2 between two layers are 3.46 and $4.02 \stackrel{\circ}{A}$, respectively. Therefore the distances are larger than in graphite stacking and we expect a weak interaction between the two GNFs. By careful examination of bilayer GNFs, we do not found that the interatomic distance in different molecules is closer than this value. The HOMO-LUMO and DOS are shown in Figs. 11(g,h) and Figs. 12(b), respectively. There is no mixing in the frontier orbital. The energy is slightly higher than in the case with $\theta=0^{\circ}$. Note that if the GNFs does not bent the mutual interaction energy of $\theta=180^{\circ}$ could be lower than $\theta=0^{\circ}$. The energy gap is found to be $0.82 \mathrm{eV}$ which is less than for the $\theta=0^{\circ}$ case. This is due to the fact that the bilayer GNFs with $\theta=180^{\circ}$ has a larger net dipole and a different orientation as compared to the previous case.

iii) $\theta=90^{\circ}$ : The tail of the top GNF is directed towards the head and away from the tail of the bottom GNF. After optimization the mutual angle is not $\theta=90^{\circ}$ and there is an additional mutual rotation. The closest and longest distances between different layers (not including hydrogen atoms) thus appear in the vicinity of these head-tail and tail-tail, are 3.35 and $4.89 \stackrel{\AA}{A}$, respectively, which are equal and longer than the graphite stacking distance, respectively. The HOMO-LUMO and DOS are shown in Figs. 11(k,l) and Figs. 12 (c), respectively. There is no mixed frontier orbitals and the energy is larger than those for $\theta=0^{\circ}$ and $\theta=180^{\circ}$. The net dipole is much larger than those of $\theta=0^{\circ}$ and $\theta=180^{\circ}$ resulting to a lower energy gap, i.e. $0.41 \mathrm{eV}$. Note that for two GNFs consisting of two $\mathrm{C}_{40} \mathrm{~F}_{16}$ our calculation was not fully optimized, hence $\theta=180^{\circ}$ is not likely the preferential bilayer structure for this GNFs.

Based on the above results, we may reasonably regard that for a large-polar GNFs such as $\mathrm{C}_{40} \mathrm{H}_{16}$, the polarity of GNFs determines the mutual orientation of the GNFs arrangement in the crystal structure. Additionally, it is interesting to note that while a single $\mathrm{C}_{40} \mathrm{H}_{16}$ is planer, however it bends when it interact with other GNFs which is due to its finite size (the edges interact). The resulting bilayer structures depend on the type of stacking and there is a tilting of the flakes.

\section{RAMAN SPECTROSCOPY}

Raman spectroscopy $\sqrt[30]{30}$ is a non-destructive and quick characterization technique which gives structural and electronic information. For graphene nano-flakes, the most intense 
TABLE II. Net dipole moment, energy gap and cohesive energy for bilayer GNFs with different mutual orientation

\begin{tabular}{l|ccc}
\hline & $\mathrm{P}($ Debye $)$ Gap $(\mathrm{eV}) \frac{\text { Energy }}{N_{C}+N_{H, F}}(\mathrm{eV} /$ atom $)$ \\
\hline$\theta=0^{\circ}$ & & & \\
$\mathrm{C}_{40} \mathrm{H}_{16}$ & 0.006 & 0.99 & -7.012 \\
$\mathrm{C}_{40} \mathrm{~F}_{16}$ & 0.781 & 0.90 & -6.905 \\
\hline$\theta=180^{\circ}$ & & & \\
$\mathrm{C}_{40} \mathrm{H}_{16}$ & 0.059 & 0.82 & -7.010 \\
$\mathrm{C}_{40} \mathrm{~F}_{16}$ & - & - & - \\
\hline$\theta=90^{\circ}$ & & & \\
$\mathrm{C}_{40} \mathrm{H}_{16}$ & 0.999 & 0.41 & -7.008 \\
$\mathrm{C}_{40} \mathrm{~F}_{16}$ & 1.977 & 0.38 & -6.904 \\
\hline
\end{tabular}

peaks are $\mathrm{G}$ and $\mathrm{D}$, appearing around $1585 \mathrm{~cm}^{-1}$ and $1350 \mathrm{~cm}^{-1}$, respectively. The $\mathrm{G}$ peak corresponds to the $\mathrm{C}-\mathrm{C}$ bond stretching, i.e. the first order Raman-allowed $\mathrm{E}_{2 g}$ phonon at the Brillouin zone center. The D peak requires a defect to be activated. Density functional calculations using the hybrid functional B3LYP with polarized basis set $6-311 \mathrm{~g}^{* *}$ have been carried out in order to obtain the Raman spectrum (we used standard method for scaling of the frequencies which has been adopted in GAUSSIAN09 software).

Raman spectra are calculated for some typical H- and F- passivated nano-flakes (as shown in Fig. 1) and bilayer GNFs. We notice that the introduction of a pentagon in the $\mathrm{C}_{41} \mathrm{H}_{15}$ nano-flake introduces more high frequency modes beyond the G-peak as compared to nondefective nano-flake (e.g. $\mathrm{C}_{50} \mathrm{H}_{18}$ as shown in Fig. $14(\mathrm{~b})$ ), which is due to the fact that modes localize around the defect (as shown in Fig. 13). Introduction of a pentagon in the $\mathrm{C}_{41} \mathrm{~F}_{15}$ also introduces more high frequency modes similar to H-pasivated clusters. Furthermore, a prominent feature at $1260 \mathrm{~cm}^{-1}$ characteristic of covalent $\mathrm{C}-\mathrm{F}$ bond stretching ${ }^{31}$ has been observed. D peak can be found in all GNFs due to the finite crystalline size where the edges of the nano-flakes can be seen as defects. The ratio of the intensities of D and G peak increases as the nano-flake size decreases due to the relative increase of the number of edge atoms and the less ordered crystalline structure in smaller GNFs (see Fig. 14(a)). For highly symmetric nano-flakes, e.g. $\mathrm{C}_{24} \mathrm{H}_{12}$ only $\mathrm{G}$ - and D-peaks are dominant. A localized mode 


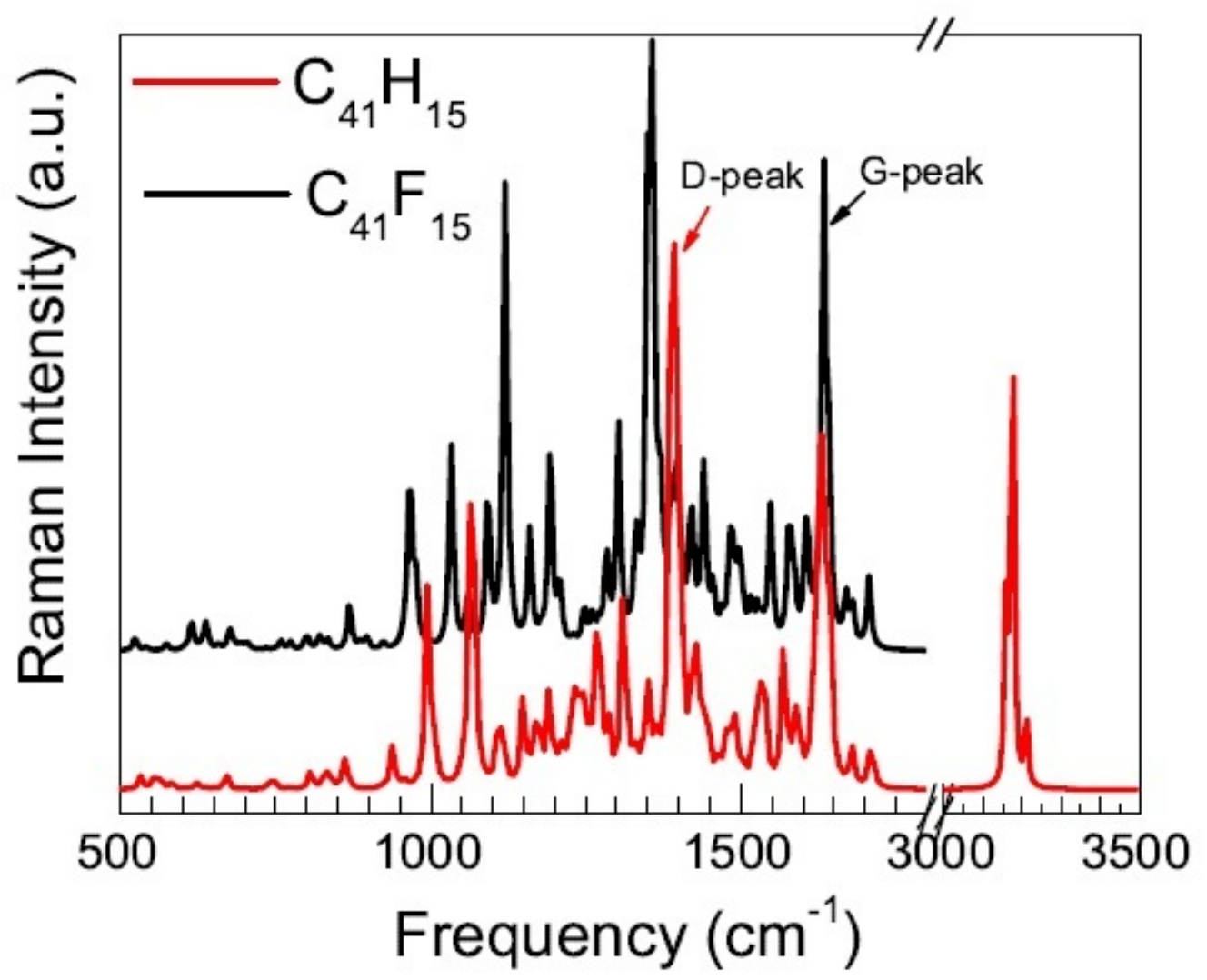

FIG. 13. (Color online) Raman spectrum of $\mathrm{C}_{41} \mathrm{H}_{15}$ and $\mathrm{C}_{41} \mathrm{~F}_{15}$ nano-flakes.

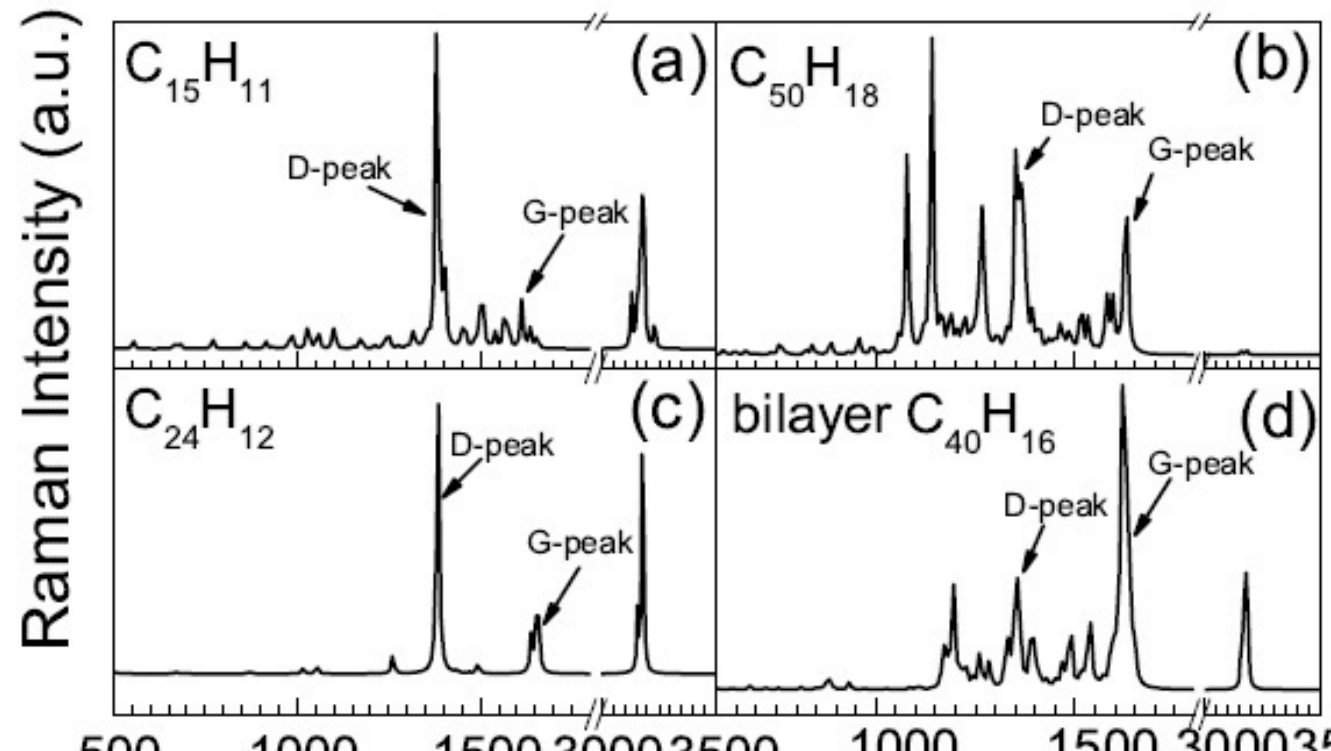

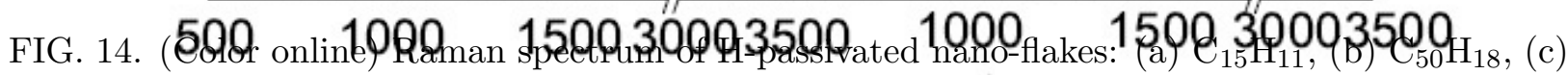
$\mathrm{C}_{24} \mathrm{H}_{12}$, and (d) bilayer $\mathrm{C}_{40} \mathrm{H}_{16}$. Frequency $\left(\mathrm{cm}^{-1}\right)$ 
is found to appear at $\approx 3100 \mathrm{~cm}^{-1}$ in H-passivated GNFs, which is the typical vibrational mode of the $\mathrm{C}-\mathrm{H}$ bond ${ }^{32}$.

For a parallel bilayer GNFs, broad G- and D- bands appear (Fig. 14(d)). We also found that the intensity of the Raman spectra in GNFs are different than those experimentally reported for graphen ${ }^{30}$. Firstly, because of the edge effects which causes non-uniform distribution of C-C bonds over the GNFs and secondly because of the large ratio between the number of defects and the total number of carbon atoms in each particular GNF. These enhance the intensity of D peak with respect to G peak while they are not shifted with respect to graphene.

\section{CONCLUSION}

Using extensive ab-initio calculations we studied the electronic properties of GNFs with several different number of carbon atoms and two different atoms for edge termination. The n-fold symmetry causes no net dipole in GNFs. Breaking the n-fold symmetry by heptagon and pentagon defects and reducing the symmetries to mirror symmetry enhances the polarization. We found that the larger the dipole moment the lower the energy gap for both type of saturated atoms. The cohesive energy of GNFs reduces with increasing carbon atoms for constant number of passived atoms. On average the energy gap decreases rapidly. The electrostatic potential around GNFs control both the polarization and the energy gap of the GNFs. Our spin polarized calculations show that the difference between the energy gap of up and down spin is maximized mostly for GNFs with pentagon (and heptagon) defects. The H-passivated GNFs have a larger difference between the spin-up and spin-down energy gap as compared to F-passivated GNFs. The bilayer GNFs reveal a clear dipole-dipole interaction which is a consequence of the mutual orientation between the permanent dipoles in the system. The bilayer GNFs are not necessarily planar structures and may have a curved structure.

ACKNOWLEDGMENTS This work was supported by the EU-Marie Curie IIF postdoc Fellowship/299855 (for M.N.-A.), the ESF-EuroGRAPHENE project CONGRAN, the Flemish Science Foundation (FWO-Vl), and the Methusalem Foundation of the Flemish Government. 


\section{REFERENCES}

${ }^{1}$ D. Abergel, V. Apalkov, J. Berashevich, K. Zieler, and T. Chakraborty, Adv. Phys. 59, $261(2010)$.

${ }^{2}$ A. Castro Neto, F. Guinea, N. Peres, K. Novoselov, and A. Geim, Rev. Mod. Phys. 81, 109 (2009).

${ }^{3}$ Y.-W. Son, M. L. Cohen, and S. G. Louie, Nature (London) 444, 347 ( 2006).

${ }^{4}$ J. Wu, W. Pisula, and K Mullen, Chem. Rev. 107, 718 (2007).

${ }^{5}$ L. Zhi and K. Mullens, J. Mater. Chem. 18, 1472 (2008).

${ }^{6}$ K. S. Novoselov, A. K. Geim, S. V. Morozov, D. Jiang, Y. Zhang, S. V. Dubonos, I. V. Grigorieva, and A. A. Firsov, Science 306, 666 (2004).

${ }^{7}$ S. Park and R. Ruoff, Nature Nanotechnology 4, 217 (2009).

${ }^{8}$ M. Terrones, Nature (London) 458, 845 (2009).

${ }^{9}$ P. Potasz, A. D. Güçlü, O. Voznyy, J. A. Folk, and P. Hawrylak, Phys. Rev. B 83, 174441 (2011).

${ }^{10}$ K. Chang and F. M. Peeters, Phys. Rev. B 68, 205320 (2003).

${ }^{11}$ Y. -W. Son, M. L. Cohen, and S. G. Louie, Phys. Rev. Lett 97, 216803 (2006).

${ }^{12}$ M. Ezawa, Phys. Rev. B 73, 045432 (2006); L. Brey and H. A. Fertig, Phys. Rev. B 73, 235411 (2006); K.-I. Sasaki, S. Murakami, and R. Saito, J. Phys. Soc. Jpn. 75, 074713 (2006); S. Okada and A. Oshiyama, Phys. Rev. Lett. 87, 146803 (2001); H. Lee, Y. -W. Son, N. Park, S. Han, and J. Yu, Phys. Rev. B 72, 174431 (2005); D. A. Abanin, P. A. Lee, L. S. Levitov, Phys. Rev. Lett. 96, 176803 (2006); Y.-W. Son, M. L. Cohen, and S. G. Louie, Nature (London) 444, 347 ( 2006).

${ }^{13}$ A. D. Güçlü, P. Potasz, and P. Hawrylak, Phys. Rev. B 82, 155445 (2010).

${ }^{14}$ X. Yan, X. Cui, B. Li, and L.-s. Li, Nano Lett. 10, 1869 (2010).

${ }^{15}$ H. Zheng and W. Duley, Phys. Rev. B 78, 045421 (2008).

${ }^{16}$ W. L. Wang, S. Meng, and E. Kaxiras, Nano Lett. 8, 241 (2008); O. V. Yazyev, W. L. Lang, S. Meng, and E. Kaxiras, Nano Lett. 8, 766 (2008).

${ }^{17}$ O. Voznyy, A. D. Güçlü, P. Potasz, and P. Hawrylak, Phys. Rev. B 83, 165417 (2011).

${ }^{18}$ I. Snook and A. Barnard, Physics and applications of graphene-theory, edited by Sergey Mikhailov, (InTech, Croatia, 2011), Chap. 13.

${ }^{19}$ A. Kuc and T. Heine, and G. Seifert, Phys. Rev. B 81, 085430 (2010). 
${ }^{20}$ S. K. Singh, M. Neek-Amal, and F. M. Peeters, Phys. Rev. B 87, 134103 (2013).

${ }^{21}$ D. P. Kosimov, A. A. Dzhurakhalov, and F. M. Peeters, Phys. Rev. B 81, 195414 (2010).

${ }^{22}$ A. D. Güçlü, P. Potasz, and P. Hawrylak, Phys. Rev. B 84, 035425 (2011).

${ }^{23}$ M. J. Frisch et al., GAUSSIAN 09, Revision A.1, Gaussian, Inc., Wallingford CT, 2009.

${ }^{24}$ A. D. Becke, J. Chem. Phys. 98, 5648 (1993).

${ }^{25}$ E. Rudberg, P. Salek, and Y. Luo, Nano Lett. 7, 2211 (2007).

${ }^{26}$ M. A. Akhukov, A. Fasolino, Y. N. Gornostyrev, and M. I. Katsnelson, Phys. Rev. B 85, 115407 (2012).

${ }^{27}$ Z. Z. Zhang, Kai Chang, and F. M. Peeters, Phys. Rev. B 77, 235411 (2008).

${ }^{28}$ A. K. Geim and K. S. Novoselov, Nature Mater. 6, 183 (2007).

${ }^{29}$ K. A. Ritter and J. W. Lyding, Nature Mater. 8, 235 (2009).

${ }^{30}$ A. C. Ferrari and D. M. Basko, Nat. Nano. 8, 235 (2013); M. S. Dresselhaus and P. C. Eklund, Adv. Phys. 49, 705 (2000); S. Reich and C. Thomsen, Philos. Trans. R. Soc. London, Ser. A 362, 2271 (2004).

${ }^{31}$ S. B. Bon, L. Valentini, R. Verdejo, J. L. G. Fierro, L. Peponi, M. A. Lopez-manchado, and J. M. Kenny, Chem. Mater. 21, 3433 (2009).

${ }^{32}$ J. Demaison and G. Wlodarczak, Struct. Chem. 5, 57 (1992). 